\title{
A enfermagem no contexto da reprodução assistida: uma revisão integrativa da
}

\section{literatura}

\author{
Nursing in the context of assisted reproduction: an integrative literature review \\ Enfermería em el contexto de reproducción asistida: revisión integrativa de la literatura
}

\author{
Ranyelle Raynara Farias Valadares \\ ORCID: https://orcid.org/0000-0002-3692-1771 \\ Universidade Paulista, Brasil \\ E-mail: ranyelleraynara@ hotmail.com \\ Luciana Aparecida Marchiori Thimoteo Alves \\ ORCID: https://orcid.org/0000-0002-4242-4034 \\ Universidade Paulista, Brasil \\ E-mail: marchiori2011@gmail.com \\ Maria Luiza Rêgo Bezerra \\ ORCID: https://orcid.org/0000-0002-3336-7760 \\ Universidade Paulista, Brasil \\ E-mail: maria.bezerra@docente.unip.br
}

\begin{abstract}
Resumo
Objetivos: abordar o papel e as ações da enfermagem na reprodução humana, sua importância no suporte psicológico e técnico dos pacientes que passam pelas etapas e procedimentos da RHA. Métodos: trata-se de um estudo de revisão integrativa da literatura com abordagem qualitativa e exploratória voltada para a importância do papel e ações dos enfermeiros na RHA. Resultados: O papel da enfermagem na Reprodução Humana Assistida ultrapassa a subjetividade técnica e traz aspectos humanos, interpessoais e de cuidado no envolvimento de todo o processo, por ser o profissional que passa mais tempo em contato com o paciente, este deve promover a assistência necessária para o casal, sendo o principal canal entre os pacientes e o especialista, o enfermeiro orienta, prepara e participa ativamente das técnicas da RHA. Conclusão: o enfermeiro deve demonstrar capacitação e conhecimento teórico envolto de constantes pesquisas e atualizações, proporcionar a seus pacientes acolhimentos, atenção, motivação e respeito no tratamento de infertilidade em seres humanos, sendo que a equipe de enfermagem é o canal direto e rápido entre pacientes e especialistas em todo o processo de RHA.
\end{abstract}

Palavras-chave: Reprodução; Técnicas de reprodução assistida; Enfermagem; Fiv.

\begin{abstract}
Objectives: to address the role and actions of nursing in human reproduction its importance in the psychological and technical support of patients who go through the stages and procedures of AHR. Methods: this is an integrative literature review study with a qualitative and exploratory approach focused on the importance of the role and actions of nurses in AHR. Results: the role of nursing in Assisted Human Reproduction goes beyond technical subjectivity and brings human, interpersonal and care aspects in the involvement of the entire process, as the professional who spends more time in contact with the patient, he/she must provide the necessary assistance for the couple, being the main channel between the patients and the specialist, the nurse guides, prepares and actively participates in the techniques of AHR. Conclusion: nurses must demonstrate training and theoretical knowledge involved in constant research and updates, provide their patients with care, attention, motivation and respect in the treatment of infertility in human beings, with the nursing team being the direct and fast channel between patients and experts in the entire RHA process.
\end{abstract}

Keywords: Reproduction; Assisted reproduction techniques; Nursing; Fiv.

\section{Resumen}

Objetivos: abordar el papel y las acciones de la enfermaría em la reproducción humana, su importancia en el acompañamiento psicológico y técnico de los pacientes que atraviesan las etapas y procedimientos de AHR. Métodos: se trata de un estudio de revisión integradora de la literatura con abordaje cualitativo y exploratorio centrado em la importancia del papel y las acciones de las enfermeras en la AHR. Resultados: El rol de la enfermaría em la Reproducción Humana Asistida va más ala de la subjetividad técnica y aporta aspectos humanos, interpersonales y asistenciales em la implicación de todo el proceso, ya que el profesional que pasa más tiempo en contacto con el paciente, debe brindar la necesaria asistencia a la pareja, siendo el canal principal entre los pacientes y el especialista, la enfermera guía, prepara y participa activamente en las técnicas de AHR. Conclusión: el enfermero debe demostrar formación y conocimientos teóricos involucrados en la investigación y actualización constante, brindar a sus pacientes 
cuidado, atención, motivación y respeto en el tratamiento de la infertilidad en seres humanos, siendo el equipo de enfermaría el canal directo y rápido entre pacientes y expertos en todo el proceso de RHA.

Palabras clave: Reproducción; Técnica de reproducción asistida; Enfermería; Fiv.

\section{Introdução}

Com os avanços progressivos tanto medicinais quanto tecnológico, surtiram efeitos nos procedimentos medicinais, promovendo aos casais que obtiveram o diagnóstico de infertilidade, a possibilidade de conceber seu primeiro filho. A Organização Mundial da Saúde (OMS) define a infertilidade como a ausência de gestação, após 12 meses de tentativas com relações sexuais regulares sem a utilização de qualquer método contraceptivo, e também, em seu grau de complexidade podendo ser caracterizada como primária (sem gestações anteriores) ou secundária (aquela à qual ocorreu ao menos uma gestação anterior) (Leite, et al.,2021).

A infertilidade pode ter sua origem direcionada por diversos fatores, podendo estar ligadas pelo homem ou pela mulher, ou em ambos. Para as mulheres, algumas das causas podem ser: alterações hormonais, idade, endometriose, doenças ovarianas, alterações nas tubas uterinas entre outros. Para os homens, pode ocorrer: obstrução de dutos, alterações na ejaculação, alterações no esperma. Ademais, a infertilidade conjugal pode advir de fatores genéticos, ISTs, sedentarismo, obesidade, stress, uso de bebidas alcoólicas e a infertilidade inexplicável (ISCA) que mesmo após a realização de todos exames necessários para o diagnóstico, não se consegue chegar a uma conclusão exata da causa. De acordo com dados da Sociedade Brasileira de Reprodução Assistida (SBRA) aponta que, em todo o mundo cerca de 50 a 80 milhões de pessoas podem ser inférteis, no Brasil a taxa se aproxima de 8 milhões de homens e mulheres (Leite, et al.,2021).

Neste contexto a Reprodução Humana Assistida (RHA) é um conjunto de técnicas e intervenções biotecnológicas, desenvolvida com o intuito de promover a gravidez em mulheres com diagnostico de infertilidade, essas técnicas são utilizadas em países mais desenvolvidos, no Brasil ainda tem sua prevalência em âmbito privado, contudo algumas instituições universitárias ainda realizam esses procedimentos (Leite, et al.,2021).

As principais técnicas da RHA se compõem em coito programado, técnica mais simples da RHA, que define por acompanhamento do ciclo menstrual da mulher, perfazendo no melhor momento para se ocorrer a relação sexual visando uma gestação. Esta técnica consiste na inseminação artificial, consiste no deposito do sêmen previamente recolhido em laboratório no canal vaginal, cervical ou até mesmo no útero da mulher compreendendo a fertilização in vitro (FIV), técnica mais complexa que utiliza embriões selecionados e cultivados em ambiente laboratorial de forma assistida, onde ocorre a união dos gametas, mas existem também, outras técnicas utilizadas na RHA,

Os casais que se deparam com essa dificuldade podem ter experiências sentimentais de vulnerabilidade, impotência, perda de controle das suas vidas pessoais e do projeto de vida nomeadamente o objetivo de ter filhos. A problemática é encarada como uma crise podendo afetar aspectos emocionais, psíquicos, físicos e socioculturais. (Passos \& Silva, 2019; Carvalho \& Rodrigues 2021).

Em suma o trabalho buscou abordar o papel e as ações da enfermagem na reprodução humana, sua importância no suporte psicológico e técnico dos pacientes que passam pelas etapas e procedimentos da RHA. A atuação dos enfermeiros é de suma importância na reprodução humana, pois trabalham em conjunto com as demais equipes inseridas no sistema, no processo de cuidados, assim garantindo o atendimento humanizado e auxilio em consultas até o período de recuperação pósprocedimentos. 


\section{Metodologia}

Este estudo constitui uma revisão integrativa da literatura cientifica sobre A Enfermagem no contexto da RA e para o alcance do objetivo proposto, optou-se pela revisão integrativa da literatura.

A revisão integrativa da literatura é um método que permite a incorporação das evidencias na pratica clinica é fundamental em conhecimentos científicos com resultados de qualidade, este método requer a formação de um problema, a pesquisa de literatura, a avaliação crítica de um conjunto de dados e a apresentação dos resultados. Deste modo, permite reunir e sintetizar resultados de pesquisas sobre um tema delimitado ou questão de forma sistemática e ordenada, contribuindo para o aprofundamento do conhecimento do tema investigado (Sousa, Vieira, Sandy \& Antunes 2017).

A coleta de dados foi realizada em setembro de 2021, utilizando bancos de dados como Literatura Latino-Americana e do Caribe em Ciências da Saúde (LILACS), Scientific Eletronic Library Onlaine (SCIELO), Base de Dados de Enfermagem (BDENF) e Biblioteca Virtual em Saúde (BVS), utilizando os seguintes descritores e palavras chaves: Reprodução; Técnicas de reprodução assistida; Enfermagem; Fiv.

Foram elaboradas combinações entre descritores e/ou palavras chaves que originaram as estratégias de busca descritas abaixo (Quadro 1):

Quadro 1: Estratégia de busca. Brasília DF 2021.

\begin{tabular}{|c|c|}
\hline $\begin{array}{l}\text { LILACS e } \\
\text { BVS }\end{array}$ & $\begin{array}{l}\text { Reprodução AND Enfermagem; Reprodução OR Enfermagem; Técnicas de reprodução } \\
\text { Assistida; fertilização in vitro OR Enfermagem. }\end{array}$ \\
\hline SCIELO & $\begin{array}{l}\text { Reprodução AND Enfermagem; } \\
\text { Reprodução OR Enfermagem. }\end{array}$ \\
\hline BDENF & $\begin{array}{l}\text { Reprodução AND Enfermagem; } \\
\text { Enfermagem OR Fiv. }\end{array}$ \\
\hline
\end{tabular}

Fonte: Autores

A partir da coleta de dados, localizaram-se 1.056 estudos que foram submetidos a primeira etapa de avaliação, os seguintes critérios de inclusão para selecionar os artigos utilizados: classificação de estudos nas respectivas bases de dados, relação direta com os descritores, idiomas de publicação em português e inglês, artigos com relevância pesquisa que aborde a importância da enfermagem na RA e período de publicação entre 2005 a 2021. O critério de exclusão foram artigos com outros métodos de reprodução humana e artigos que não oferecem embasamento de pesquisa na importância da enfermagem na Reprodução Assistida.

Assim, obteve-se uma amostra de 35 artigos, após leituras minuciosas, focadas nos objetivos a serem alcançados foram selecionados 20 para o estudo.

A análise e a interpretação dos dados foram feitas de forma organizada por meio da visualização dos dados no fluxograma, com detalhamento da pesquisa apresentado a seguir na Figura 1. 
Figura 1: Fluxograma coleta e análise dos dados. Brasília-DF, 2021.

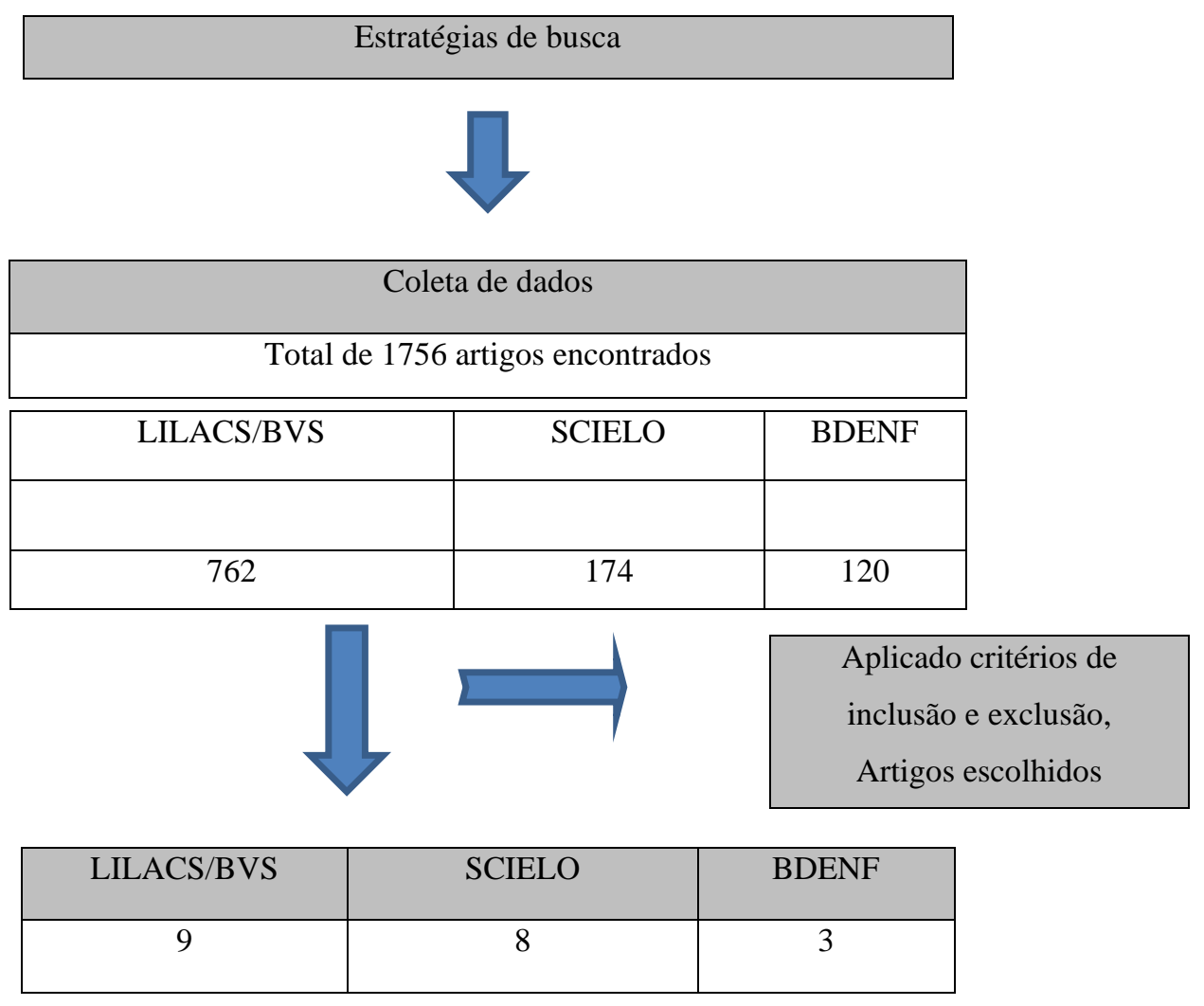

Fonte: Autores.

\section{Resultados}

Após análise, apresenta a amostra final de20 artigos selecionados, contendo as seguintes informações: Titulo, periódico, ano, método e nível de evidencia, que foram utilizados como critério de caracterização dos resultados a saber, o método de análise da temática possibilitou categorizar, interpretar e agrupar os dados, o Quadro 1 ilustra os artigos selecionado para analise na integra.

Quadro 1: Caracterização dos resultados. Brasília-DF, 2021.

\begin{tabular}{|c|c|c|c|c|}
\hline TíTULO & PERIÓDICO & ANO & MÉTODO & $\begin{array}{c}\text { NÍVEL DE } \\
\text { EVIDENCIA }\end{array}$ \\
\hline $\begin{array}{c}\text { Enfermagem e o planejamento } \\
\text { familiar: as interfaces da contracepção }\end{array}$ & $\begin{array}{l}\text { Revista brasileira } \\
\text { de enfermagem }\end{array}$ & 2005 & Pesquisa qualitativa & 4 \\
\hline $\begin{array}{c}\text { Revisão integrativa: método de } \\
\text { pesquisa para incorporação de } \\
\text { evidencias na saúde e na enfermagem }\end{array}$ & $\begin{array}{l}\text { Texto e contexto } \\
\text { enfermagem }\end{array}$ & 2009 & $\begin{array}{l}\text { Revisão integrativa do } \\
\text { estudo }\end{array}$ & 6 \\
\hline $\begin{array}{l}\text { Procedimentos de fertilização in vitro: } \\
\text { experiência de mulheres e homens }\end{array}$ & $\begin{array}{l}\text { Psicologia em } \\
\quad \text { estudo }\end{array}$ & 2010 & Revisão bibliográfica & 1 \\
\hline $\begin{array}{l}\text { Estratégia educativa voltada para } \\
\text { enfermeiros sore atenção básica a } \\
\text { infertilidade: estudo de intervenção }\end{array}$ & $\begin{array}{l}\text { Revista gaúcha de } \\
\text { enfermagem }\end{array}$ & 2012 & $\begin{array}{c}\text { Revisão integrativa do } \\
\text { estudo }\end{array}$ & 2 \\
\hline $\begin{array}{l}\text { Efeitos da infertilidade no } \\
\text { relacionamento dos cônjuges }\end{array}$ & $\begin{array}{l}\text { Revista da Escola } \\
\text { de Enfermagem da } \\
\text { USP }\end{array}$ & 2012 & $\begin{array}{c}\text { Pesquisa qualitativa, } \\
\text { descritiva e exploratória, } \\
\text { com analise de conteúdo }\end{array}$ & 4 \\
\hline Bioética e reprodução humana & $\begin{array}{l}\text { Revista de saúde } \\
\text { coletiva }\end{array}$ & 2014 & Estudo descritivo & 3 \\
\hline
\end{tabular}




\begin{tabular}{|c|c|c|c|c|}
\hline $\begin{array}{l}\text { Panorama biótico e jurídico da } \\
\text { reprodução humana assistida no Brasil }\end{array}$ & $\begin{array}{c}\text { Revista de bioética } \\
\text { y Derecho }\end{array}$ & 2015 & Qualitativa & 2 \\
\hline $\begin{array}{l}\text { Representações sociais de enfermeiros } \\
\text { da área da saúde sexual e reprodutivas } \\
\text { sobre reprodução humana assistida }\end{array}$ & $\begin{array}{l}\text { Base de dados } \\
\text { internacionais }\end{array}$ & 2015 & $\begin{array}{l}\text { Pesquisa qualitativa/saúde } \\
\text { sexual e reprodutiva }\end{array}$ & 4 \\
\hline $\begin{array}{c}\text { Perspectiva de casais em relação à } \\
\text { infertilidade e reprodução assistida: } \\
\text { uma revisão sistemática }\end{array}$ & $\begin{array}{l}\text { Reprodução e } \\
\text { climatério }\end{array}$ & 2016 & $\begin{array}{l}\text { Revisão integrativa do } \\
\text { estudo }\end{array}$ & 5 \\
\hline $\begin{array}{c}\text { Metodologia de Revisão Integrativa da } \\
\text { Literatura em Enfermagem }\end{array}$ & $\begin{array}{c}\text { Revista integrativa } \\
\text { de enfermagem }\end{array}$ & 2017 & Pesquisa qualitativa & 6 \\
\hline $\begin{array}{l}\text { Enfermagem e reprodução humana: } \\
\text { uma associação para a vida }\end{array}$ & Repositório Faema & 2018 & $\begin{array}{l}\text { Pesquisa qualitativa usando } \\
\text { a análise de conteúdo }\end{array}$ & 3 \\
\hline $\begin{array}{c}\text { O estado da arte da atuação da } \\
\text { enfermagem na reprodução assistida. }\end{array}$ & $\begin{array}{l}\text { A enfermagem e a } \\
\text { reprodução } \\
\text { humana. }\end{array}$ & 2018 & Estudo descritivo & 2 \\
\hline $\begin{array}{l}\text { Analise critica sobre a evolução das } \\
\text { normas éticas para a utilização das } \\
\text { técnicas de reprodução assistida no } \\
\text { Brasil }\end{array}$ & $\begin{array}{l}\text { Ciência e Saúde } \\
\text { Coletiva }\end{array}$ & 2019 & $\begin{array}{l}\text { Qualitativo, exploratório e } \\
\text { descritivo }\end{array}$ & 2 \\
\hline $\begin{array}{c}\text { Infertilidade e inseminação artificial } \\
\text { no século XVI }\end{array}$ & $\begin{array}{l}\text { Centúrias de Curas } \\
\text { Medicinais }\end{array}$ & 2019 & Estudo descritivo & 5 \\
\hline $\begin{array}{c}\text { Fatores que interferem no sucesso da } \\
\text { reprodução humana assistida e o } \\
\text { envolvimento do enfermeiro no } \\
\text { processo }\end{array}$ & $\begin{array}{l}\text { Revista JRG de } \\
\text { Estudos } \\
\text { Acadêmicos }\end{array}$ & 2019 & $\begin{array}{l}\text { Pesquisa qualitativa, } \\
\text { descritiva e exploratória, } \\
\text { com analise de conteúdo }\end{array}$ & 5 \\
\hline $\begin{array}{c}\text { Trabalho do enfermeiro em reprodução } \\
\text { humana assistida: entre tecnologia e } \\
\text { humanização }\end{array}$ & $\begin{array}{l}\text { Revista Brasileira } \\
\text { de Enfermagem }\end{array}$ & 2020 & $\begin{array}{c}\text { Exploratório descritivo, } \\
\text { com abordagem qualitativa } \\
\text { do tipo análise de discurso. }\end{array}$ & 1 \\
\hline $\begin{array}{c}\text { Estratégia saúde da família e a } \\
\text { integralidade do cuidado: percepção } \\
\text { dos profissionais }\end{array}$ & $\begin{array}{l}\text { Revista baiana de } \\
\text { enfermagem }\end{array}$ & 2020 & $\begin{array}{c}\text { Revisão integrativa do } \\
\text { estudo }\end{array}$ & 4 \\
\hline $\begin{array}{l}\text { Aspecto emocional e reprodução } \\
\text { humana assistida: uma revisão } \\
\text { integrativa da literatura }\end{array}$ & $\begin{array}{l}\text { Femina artigo de } \\
\text { revisão }\end{array}$ & 2021 & Qualitativo, descritivo & 1 \\
\hline $\begin{array}{c}\text { O vivido da reprodução do casal } \\
\text { heterossexual sorodiscordante para o } \\
\text { HIV: Possibilidades para o cuidado } \\
\text { em enfermagem e na saúde } \\
\text { reprodutiva }\end{array}$ & $\begin{array}{l}\text { Universidade } \\
\text { Federal de Santa } \\
\text { Catarina }\end{array}$ & 2021 & $\begin{array}{l}\text { Exploratório descritivo, } \\
\text { com abordagem qualitativa } \\
\text { do tipo análise de discurso }\end{array}$ & 7 \\
\hline $\begin{array}{l}\text { Prematuridade em gestação resultantes } \\
\text { de fertilização IN VITRO }\end{array}$ & $\begin{array}{l}\text { Repositório digital } \\
\text { unicesumar }\end{array}$ & 2021 & Revisão bibliográfica & 3 \\
\hline
\end{tabular}

Fonte: Autores.

\subsection{Discussão}

\subsubsection{Histórias da reprodução humana assistida}

O rompimento de antigos estilos de vida, hábitos e padrões impulsionados principalmente por avanços tecnológicos e científicos provocaram grandes transformações na humanidade. A reprodução humana foi e continua sendo um assunto relevante para o ser o humano, passar o seu legado para frente, a sua linhagem familiar, era um aspecto primordial para as sociedades em geral, sendo assim, a infertilidade era e continua sendo um assunto delicado e que causa sofrimento para casais e principalmente para as mulheres.

Os primeiros relatos que envolvem a preocupação com a fertilidade, ocorreram cerca de 2.200 a.C., com rituais de fecundidade utilizando diversas ferramentas como estatuas, animais, árvores, algumas religiões acreditavam que a infertilidade seria um castigo divino, já na Idade Média, utilizavam-se critérios de composição corporal como o fator da mulher ser muito 
magra, debilitada ou muito gorda para justificar a dificuldade em engravidar, para os homens diziam que os testículos eram frios ou secos e que não tinham força suficiente para engravidar a mulher (virilidade) (Carvalho \& Rodrigues 2019; Leite, Morais, Leite A \& Guimaraes 2018).

Classificado pela Sociedade Brasileira de Reprodução Humana (SBRH) a RHA é um "conjunto de técnicas onde uma equipe multidisciplinar tem participação estreita no acompanhamento do desenvolvimento folicular, detecção e indução da postura ovular, facilitação ao mesmo e a realização do encontro dos gametas assim como na otimização da fase lútea", essa equipe pode ser integrada por profissionais da saúde de diversos segmentos como ginecologistas, urologistas, psicólogos, biólogos, enfermeiros, biomédicos e outros, estes atuam de forma conjunta para oferecer o melhor tratamento para os casais inférteis ( Leite, Morais, Leite A \& Guimaraes 2018; Naves \& Sá2015).

Compreender a demanda por biotecnologias como RHA é se basear principalmente na questão da importância sociocultural da sociedade, em que a construção familiar amparada pela vinda de um filho biológico (descendência) se concretizava em sonho reprodutivo, mais que a realização de somente um aspecto pessoal, também como um ideal na constituição de uma família (Mohamed 2015; Leite, Morais, Leite A \& Guimaraes 2018).

Sentimentos como incapacidade, masculinidade e virilidade fizeram com que mais casais procurassem alternativas que solucionassem essa necessidade de procriação, a questão da infertilidade se observa como um grande fator de sensibilidade tanto para o homem quanto para a mulher, tendo diversas consequências psicossociais, emocionais e socioculturais (Mohamed 2015; Leite, Morais, Leite A \& Guimaraes 2018).

A Reprodução Humana Assistida (RHA) teve seu início no final da década de 70 a qual trouxe diversos avanços tecnológicos e de biotecnologia quando um grupo de pesquisadores da Austrália trouxe a ideia de se utilizar óvulos de animais para realizar uma possível fertilização em laboratório e transferir os embriões para o tratamento de infertilidade de causas tubárias (Mohamed 2015; Leite, Morais, Leite A \& Guimaraes 2018).

Em meados de 1978 ocorreu o primeiro nascimento oriundo de RHA na Inglaterra, ou seja, o primeiro "bebê de proveta", logo após decorridos também na Índia e na Escócia. No EUA o primeiro bebê nascido através das técnicas de Reprodução Humana Assistida, aconteceu em 1981 pelo método de Fertilização In Vitro, no Brasil a primeira RHA aconteceu no ano de 1984, pela qual o país se tornou o primeiro, na América latina, a realizar um procedimento de Reprodução Humana, tornando um grande marco histórico da RHA (Mohamed 2015; Leite2019)

Com isso a RHA trouxe esperanças a esses casais, que envolve comprometimento tanto do casal quanto dos profissionais envolvidos, pois esse processo demanda várias etapas que são progressivamente mais complexas e especificas. Hoje as técnicas da Reprodução Humana Assistida contemplam muito mais do que a reprodução heterossexual, mas também traz possibilidades de promover gravidez em pessoas celibatárias, casais soro discordantes do HIV, relações homoafetivas e a maternidade tardia (Mohamed 2015; Félis \& Almeida 2016; Langendorf 2015).

As técnicas de reprodução assistida que podem ser classificas como baixa e alta complexidade, levam a uma solução de problemas para casais que por via de regra, não teriam opção de tratamento, assim algumas delas são inseminação artificial (IA), fertilização in vitro (FIV), injeção intracitoplasmática de espermatozoides (ICSI), correção medicamentosa de distúrbios hormonais, tratamento de ISTs, intervenções cirúrgicas como reversão de vasectomia transferência de embriões e injeção intracitoplasmática de espermatozoides (ICSI), entre outros(Silva \& Rocha2020).

Esses processos envolvidos nas diversas técnicas da RHA podem se tornar muito desgastantes e desconfortáveis, com grande sofrimento físico e psicológico principalmente pela chance de insucesso, a qual pode se tornar uma quebra de expectativas pelo casal. As ingestões de hormônios para induzir a ovulação, a coleta de amostras, ultrassonografias, exames laboratoriais, anestesias e etc. torna o processo além de muito doloroso, caro, o que dificulta o acesso da população em geral, por conta de todos esses desgastes se faz necessário uma boa equipe multiprofissional envolvida no tratamento, que devem oferecer todas 
informações e esclarecer dúvidas para que esses casais possam ter um bom suporte psicológico para lidar com toda essa situação e que já estão enfrentando diversas dificuldades que muitas vezes já vem de um processo de tentativas frustradas, ressaltando o trabalho da enfermagem em todo esse processo pois é com esses profissionais que os pacientes tem o primeiro contato (a entrevista) quando busca tratamento em clinicas de infertilidade(Félis \& Almeida 2016; Leite, Morais, Leite A \& Guimaraes 2018).

Quando um casal decide planejar o nascimento de um filho, várias medidas são tomadas como interrupção de anticoncepcionais e a relação sexual sem proteção, porém cerca de $10 \%$ desses casais falham no primeiro ano na concepção de um bebê, nos últimos anos a procura por clinicas de infertilidade provou que esse campo representa um trabalho importante para o cuidar da enfermagem (Leite, Morais, Leite A \& Guimaraes 2018; Faria, Grieco \& Barros 2012)

Observa-se que o entendimento da enfermagem acerca de questões relacionadas a sexualidade mudou através do tempo sendo que primeiramente estava relacionado a questões como prevenção de ISTs e controle de gravidez na adolescência, após isso em questões relacionadas ao ato sexual em si e, por conseguinte nos dias atuais se baseia no entendimento mais geral e completo a respeito de sexualidade e saúde reprodutiva. E com isso entendemos que o profissional enfermeiro ainda está de certa maneira distanciado de questões relacionadas à RHA mesmo tendo um papel fundamental nesse processo (Mohamed2015; Dias 2012; Coelho2005).

A enfermagem é de suma importância na RHA sendo a porta de entrada da assistência da RHA contribuindo na ajuda dos casais no entendimento de todo o processo dando informações técnicas e auxiliando em intervenções importantes, explicando a anatomia e fisiologia da reprodução, no suporte emocional, auxiliando em diferenças culturais e apoiando nas expectativas dos casais.

O papel da enfermagem na Reprodução Humana Assistida ultrapassa a subjetividade técnica e traz aspectos humanos, interpessoais e de cuidado no envolvimento de todo o processo, por ser o profissional que passa mais tempo em contato com o paciente, este deve promover a assistência necessária para o casal, sendo o principal canal entre os pacientes e o especialista, o enfermeiro orienta, prepara e participa ativamente das técnicas da RHA, esse trabalho é fundamental para garantir a adesão ao tratamento e também de suporte as dúvidas e questionamentos que surgirão dos pacientes, gerando confiança em todo o processo, podendo aliviar através desse trabalho o estresse, ansiedade, desconforto e tensão dos procedimentos.

\subsection{Aspectos Bioéticos}

Sendo um novo campo de desenvolvimento biotecnológico a RHA trouxe diversos questionamentos pelo fato da sua interferência na vida e reprodução humana, a "criação" do primeiro bebê de proveta provocou uma ruptura na perspectiva dos processos reprodutivos humanos trazendo com si um grande debate bioético, por estar inserido principalmente em uma pluralidade de contextos como: "barrigas de aluguel (atualmente úteros de substituição), clonagem, células-tronco e criopreservação de gametas/embriões" fomentando questionamentos na sociedade correlacionada com questões mercadológicas, cientificas ou até fictícias(Mohamed2015; Braz 2005).

Inicialmente a RHA tinha como objetivo tratar problemas de cunho reprodutivo e de infertilidade, mas atualmente as técnicas atendem os mais diversos desejos reprodutivos da sociedade, que se adequou as novas demandas da sociedade e com isso trouxe sérios dilemas éticos, morais e religiosos (Mohamed2015; Faria, Grieco \& Barros 2012).

As principais preocupações acerca da RHA estão relacionadas com riscos envolvendo a saúde física e mental da mulher como a hiper estimulação hormonal ovariana, a elevada taxa de partos múltiplos e a ansiedade e a frustração com tentativas fracassadas e também da saúde dos bebês oriundos da RHA. Outra preocupação importante é com questões bioéticas que envolvem intenções e interesses acerca de embriões congelados em centros de reprodução, além disso, novas configurações de 
noções de família e o compartilhamento de material genético doado e até a utilização de embriões post mortem (Mohamed 2015; Marciano \& Amaral 2021).

Contudo, observa que dentro da esfera do Sistema Único de Saúde (SUS) o conhecimento a respeito por parte dos profissionais de enfermagem sobre a RHA parecia um tanto quanto baseada no senso comum fugindo de aspectos técnicos, baseando-se principalmente na lógica de quem utiliza do serviço público de saúde não teria, por questões financeiras, condições de custear o tratamento assim desestimulando a busca para tratamento de infertilidade e sinalizando que existem outras prioridades de custeamento dentro da saúde pública. Mas ela afirma também que esse pensamento não é de unanimidade e que muitos enfermeiros acolhiam sim esses pacientes dando à importância necessária as demandas destes mesmo que o sistema público não disponha de recursos para auxilia-los (Mohamed 2015).

A participação ativa da enfermagem na Reprodução Humana Assistida começou em meados de 1989 pela National Association of Obstetric Gynecologicand Neonatal Nurses, no Brasil esse a especialização começou em 1996 na Universidade de São Paulo, a reprodução humana é uma área da enfermagem regulamentada, mas que possui pouco incentivo.

Diante desse contexto são poucas as universidades que trazem conteúdos sobre a Reprodução Humana Assistida, enfermeiros que atuam nessa área da biotecnologia afirmam que tiveram que buscar por conta própria, fazer especializações ou até mesmo ganhar experiência somente quando se trabalha com RHA para se integrar a equipe e poder realizar um trabalho acolhedor, atencioso, responsável, ético e com o melhor método disponível (Leite Pa, Moraes, Leite A, Leite Junior \& Guimaraes 2018).

No Brasil novas tecnologias de via de regra eram introduzidas através dos meios universitários, o que não aconteceu com a RHA que até os dias atuais está predominantemente em serviços de iniciativa privada concentrando do o conhecimento nessa área ${ }^{1}$. Atualmente o acesso a saúde reprodutiva no Brasil é um grande desafio, por serem tratamentos de alto custo econômico o Estado não faz grandes investimentos nessa área, ficando de certo modo exclusivo na esfera privada e isso causa uma grande dificuldade no acesso para casais de baixa renda criando obstáculos socioculturais e de acessibilidade no cuidado ideal da saúde reprodutiva (Leite Pa, Moraes, Leite A, Leite Junior \& Guimaraes 2018).

Diante das informações apresentadas e possível observa que dentro da esfera do Sistema Único de Saúde (SUS) o conhecimento a respeito por parte dos profissionais de enfermagem sobre a RHA parecia um tanto quanto baseada no senso comum fugindo de aspectos técnicos, baseando-se principalmente na lógica de quem utiliza do serviço público de saúde não teria, por questões financeiras, condições de custear o tratamento assim desestimulando a busca para tratamento de infertilidade e sinalizando que existem outras prioridades de custeamento dentro da saúde pública. Mas ela afirma também que esse pensamento não é de unanimidade e que muitos enfermeiros acolhiam sim esses pacientes dando à importância necessária as demandas destes mesmo que o sistema público não disponha de recursos para auxilia-los (Mohamed 2015).

Grande parte das Políticas Públicas relacionadas a atenção sexual e reprodutiva partem de premissas e ações que visam principalmente a contracepção e modos de controlar a fertilidade, não contemplando aqueles em que optam pelo direito daqueles que desejam quando e quantos filhos querem ter, isso é demonstrado pelo aumento do uso de métodos contraceptivos no país nas últimas décadas, demonstrando claramente o foco das políticas de saúde pública reprodutivas, o fato de se ter essa ausência se configura como um grande impeditivo ao acesso a tecnologias reprodutivas pelos casais maias pobres ou de situação de vulnerabilidade(Mohamed2015; Souza, Rezende, Marin \& Tonhom 2020; Faria, Grieco \& Barros 2012).

$\mathrm{O}$ estudo aponta que dentro de sua pesquisa foi observado que aqueles profissionais que trabalham diretamente com RHA têm maiores percepções a respeito dessa necessidade reprodutiva e que esses tendem a compreender melhor a dificuldade/impossibilidade do processo reprodutivo de alguns casais pelas experiências vividas em seu cotidiano profissional (Leite Pa, Moraes, Leite A, Leite Junior \& Guimaraes 2018). 
Atualmente as tecnologias da RHA provam que se é possível ter soluções para as mais diversas demandas reprodutivas, representando um percurso possível para aqueles que querem manter a fertilidade como pacientes que passam por tratamentos quimioterápicos, casais soro discordantes para HIV e até mesmo casais homo afetivos e solteiros, esse ponto destaca a RHA como ponto fundamental e coloca os enfermeiros que trabalham nessa área com uma maior conexão na busca pelo filho biológico e as novas tecnologias, Queiroz (2020) destaca que de certa maneira esses agem de modo mediador ao acesso da realização das expectativas e desejos desses casais.

Aqueles profissionais que não trabalham diretamente com as tecnologias reprodutivas demonstram uma maior preocupação perante a "artificialidade" da RHA e as consequências que podem ser geradas por elas, essas preocupações são embasadas por questionamentos filosóficos e éticos, a criação de vida humana em laboratório geram diversas preocupações relacionadas principalmente com a saúde de mulheres e dos bebês, esses questionamentos muitas vezes são embasados no senso comum, pois aqueles profissionais que não trabalham diretamente com RHA demonstram pouco contato com informação técnica durante seu período acadêmico (Mendes, Silveira \& Galvão 2008)

As principais preocupações acerca da RHA estão relacionadas com riscos envolvendo a saúde física e mental da mulher como a hiper estimulação hormonal ovariana, a elevada taxa de partos múltiplos e a ansiedade e a frustração com tentativas fracassadas e também da saúde dos bebês oriundos da RHA. Outra preocupação importante é com questões bioéticas que envolvem intenções e interesses acerca de embriões congelados em centros de reprodução, além disso, novas configurações de noções de família e o compartilhamento de material genético doado e até a utilização de embriões post mortem ((Mohamed 2015).

\section{Conclusão}

A reprodução assistida é um campo em constantes progressos e avanços da ciência e tecnologia, contando com inúmeros recursos para o tratamento da infertilidade, assim baseado na revisão integrativa da literatura, os métodos apontam sua relevante ferramenta nas pesquisas e coleta de dados significativos para uma pratica baseada em evidências, ressaltando na prática onde futuros pais, além de enfrentarem a infertilidade e seus vertentes fatores psicológicos na realização do sonho de gerar uma vida, conta-se também, o fator financeiro como um recurso subjetivo no tratamento e constantes desinformações a respeito dos processos técnicas da RHA.

Mesmo sendo em uma área onde a enfermagem tem pouco acesso em sua formação a primeira coisa que se ensina e o significado de enfermagem que é a arte do cuida independente de qual área seja, o enfermeiro deve demonstrar capacitação e conhecimento teórico envolto de constantes pesquisas e atualizações, proporcionar a seus pacientes acolhimentos, atenção, motivação e respeito no tratamento de infertilidade em seres humanos, sendo que a equipe de enfermagem é o canal direto e rápido entre pacientes e especialistas em todo o processo de RHA, é com enfermeiro que o paciente tem mais contato. Pela pesquisa se constatou que o enfermeiro é responsável pelas orientações gerais de todo o tratamento, acompanhando os casais do início até o final, o mesmo atua interagindo com humanização durante todo o processo.

Desse modo, foi observado que as pesquisas, dados coletados de Literatura Latino-Americana e do Caribe em Ciências da Saúde (LILACS), Scientific Eletronic Library Onlaine (SCIELO), Base de Dados de Enfermagem (BDENF) e Biblioteca Virtual em Saúde (BVS) e pesquisas bibliográfica oriundas de artigos, fatores essenciais em que as técnicas da Reprodução Humana Assistida são de suma relevância para pesquisas contemporâneas e para uma sociedade, portanto é de caráter inovador e requer do profissional de enfermagem, constantes estudos e possíveis contribuições para o avanço da ciência e suas tecnologias na vida das pessoas, e trazendo consigo um passo para futuras gerações.

Por fim, destaca-se a importância da condução de estudos integrativo que permitam aprofundar a produção de evidências acerca dos fatores envolvidos nos processos de implementação e desempenho das ações do enfermeiro no processo de cuidar. 
Tratando-se de um sistema complexo, pesquisas com abordagem qualitativa e descritiva poderiam aprofundar o conhecimento sobre os avanços, potencialidades e dificuldades vivenciadas pelos casais.

\section{Referências}

Braz, M. (2005). Bioética e reprodução humana. Bioética e Saúde, (8)169-194 http://books.scielo.org/id/wnz6g/epub/schramm-9788575415405.epub

Carvalho, A. M. C., \& Rodrigues, I. T. (2019). Infertilidade e inseminação artificial no século XVI. História da Ciência e do Ensino construindo interfaces 20(512-522). https://revistas.pucsp.br/index.php/hcensino/article/view/44831/31020.

Coelho, E. B. S (2005/11). Enfermagem e o planejamento familiar: as interfaces da contracepção. Rev. Bras. Enferm, 58(6):66572 https://www.scielo.br/j/reben/a/BWnLvXRQt3G3HPkjPhprY7w/abstract/?lang=pt.

"Cunha, G. B. (2018). Repositório FAEMA. Enfermagem e reprodução humana: uma Associação para a vida [Internet]. 1-86. https://repositorio.faema.edu.br

Dias, A. A., Moura, E. R. F., Nogueira, P. S. F., Coutinho, J. F. V., \& Oriá, M. O. B. (2012/06). Estratégia educativa voltada para enfermeiros sobre atenção básica à infertilidade: estudo de intervenção. Revista Gaúcha de Enfermagem. 33(2):69-77 https://www.scielo.br/j/rgenf/a/rkmjX3V dsGJy8mLv6bxXcwB/?lang=pt.

Faria, D. E. P., Grieco, S. C., \& Barros, S. M. O. (2012/08). Efeitos da infertilidade no relacionamento dos cônjuges. Revista da Escola de Enfermagem da USP. 46(4):794-801 https://www.scielo.br/j/reeusp/a/qswx8tvwvT99qD3tHwzxrqJ/?lang=pt

Félis, K. C., \& Almeida, R. J. (2016) Perspectiva de casais em relação à infertilidade e reprodução assistida: uma revisão sistemática. Reprodução e Climatério (31a ed.) 105-111. https://www.sciencedirect.com/science/article/pii/S1413208716000078.

"Queiroz, A. B. A., Mohamed, R. P. S., Moura, M. A. V., Souza, I. E. O., Carvalho, M. C. M. P., \& Vieira, B. D. G. (2020). Nursing work in assisted human reproduction: between technology and humanization. Rev Bras Enferm. 73(3): e20170919. DOI: http://dx.doi.org/10.1590/0034-7167-2017-0919.

Langendorf, T. F. (2015). O vivido da reprodução do casal heterossexual sorodiscordante para o hiv: possibilidades para o cuidado em enfermagem e na saúde reprodutiva. Universidade Federal do Rio de Janeiro. Tese 156f. http://objdig.ufrj.br/51/teses/838325.pdf.

Leite, P. A., Filho, I. M. M., Félis, K. C., Leite, A.C. A., Junior, P. S. L., Guimarães, C. M. (2018). O estado da arte da atuação da enfermagem na reprodução assistida. Revista de Iniciação Científica Extensão. 1(Esp4):390-9. https://revistasfacesa.senaaires.com.br/index.php/iniciacao-cientifica/article/view/126.

Leite, T. H. (2019). Análise Crítica sobre a evolução das normas éticas para utilização das técnicas de reprodução assistida no Brasil. Ciência \& Saúde Coletiva. 24(3). https://doi.org/10.1590/1413-81232018243.30522016

Marciano, R. P., \& Amaral, W. N. (2021). Aspectos emocionais em reprodução humana assistida: uma revisão integrativa da literatura. Femina.1.49(6):379-84. https://docs.bvsalud.org/biblioref/2021/09/1290571/femina-2021-496-379-384-aspectos-emocionais-em-reproducao-huma_Wvj1JtA.pdf

Makuch, M. Y., \& Filetto, J. N. (2010). Procedimentos de fertilização in vitro: experiência de mulheres e homens. Psicologia em Estudo. 15(4):771-779. https://www.scielo.br/j/pe/a/vmczbv8kjXQCYy7n4SymVzs/?lang=pt\#.

MENDES, K. D. S., Silveira, R. C. C. P., \& Galvão, C. M. (2008). Revisão integrativa: Método de pesquisa para a incorporação de evidências na saúde e na enfermagem. Texto e Contexto - Enfermagem. 17(4): 758-64. https://doi.org/10.1590/S0104-07072008000400018

mohamed, R. P. S. (2015). Representações sociais de enfermeiros da área de saúde sexual e reprodutiva sobre reprodução humana. Universidade Federal do Rio de Janeiro. Tese 221f. https://pesquisa.bvsalud.org/portal/resource/pt/biblio-847212.

Naves, B. T. O., \& Sá, M. F. F. (2015). Panorama bioético e jurídico da reprodução humana assistida no Brasil. Rev. Bioética y Derecho. (34) 64-80. https://scielo.isciii.es/scielo.php?script=sci_arttext\&pid=S1886-58872015000200007.

Silva, G. F., Rocha, E. P., Reche, V. M., Sehn, E. G. S., Souza, V. B., Silva, F. M., \& Charlo, P. B. (2020). Prematuridade em gestações resultantes de fertilização in vitro. Global Academic Nursing Journal. 1(3):e 45. https://globalacademicnursing.com/index.php/globacadnurs/article/view/88/90

Sousa, L. M. M., Vieira, C. M., Severino, S., \& Antunes, V. (2017). A metodologia de revisão integrativa da literatura em Enfermagem. Revista investigação de enfermagem.17-26. https://www.researchgate.net/publication/321319742_Metodologia_de_Revisao_Integrativa_da_Literatura_em_Enfermagem.

Souza, A. P., Rezende, K., Marin, M. J., \& Tonhom, S. (2020). Estratégia da Família e a Integralidade do cuidado: Percepção dos profissionais. Revista Baiana de Enfermagem. 34:e34935. www.revenf.bvs.br.

Passos, M. A. N., \& Silva, N. M. (2019). Fatores que interferem no sucesso da reprodução humana assistida e o envolvimento do enfermeiro no processo1. Revista JRG De Estudos Acadêmicos, 2(5), 208-225. https://doi.org/10.5281/zenodo.4458701. 